\title{
Über die Construction der gleichseitig-hyperbolischen Schnitte der Flächen zweiten Grades.
}

\author{
Von Karl Schober in Innsbruck.
}

Das Problem, eine Wäche zweiten Grades nach einer gleichseitigen Hyperbel zu schneiden, wurde auf analytischem Wege sehon vor längerer Zeit von $S \mathrm{chl} / 0 \mathrm{mi} \mathrm{ch}^{1}$, ferner in neuester Zeit von M. Krewer ${ }^{2}$ in Dorpat und aut elementarem Wege, nur den Kegel betreffend, von $G$. Weyer ${ }^{3}$ ) in Kiel behandelt. Eine ältere Besprechung der letzteren Frage rührt von De la Chapelle ${ }^{4}$ ) her.

Einer synthetischen und constructiven Behandlung scheint jedoch die genannte Aufgabe, einige besondere Fälle ansgenommen, noch nicht unterzogen worden zu sein. Dies soll nun im Folgenden versucht werden. Die betreffenden Erörterungen und Constructionen gestalten sich infolge der Benützung einer Hilfskugelfäche überraschend einfach und durchsichtig; sie führen außjerdem zu einigen interessanten Sätzen iiber den Ort derjenigen Punkte der windschiefen Flächen zweiten Grades, in denen die beiden Erzeugenden zueinander normal sind.

Indem wir das dreiachsige Ellipsoid und das elliptische Paraboloid sammt den entsprechenden Rotationsflächen, ferner den elliptischen und den parabolischen Cylinder als Flächen, welche mit der unendlich fernen Ebene keinen Kegelschnitt gemein haben und deshalb von einer Ebene nach einer Hyperbel nicht geschnitten werden können, von vornherein aus dem Kreise unserer Erörterungen ausscheiden, haben wir uns nur mit den beiden dreiachsigen Hyper-

1) "Über die gleichseitig-hyperbolischen Schnitte der Flächen zweiten Grades". Zeitschrift f. Math. u. Physik, 6. Jahrg, pag. 418 ff.

2) , Über das Problem, eine Fläche zweiten Grades in einem der Gestalt und Größe nach gegebenen Kegelsehnitte zu schneiden". Archiv f. Math. a. Physik, II. R., 12. Thl, pag. 185 ff.

$3^{3}$ "Elementare Bestimmung der Lage der gleichseitigen Hyperbel im Kegel." Archiv f. Math. u. Physik, II. R., 14. Thl., pag. 139 ff.

4) "Traité des sections coniques", Paris 1750, deutsch von Böckmann, Karlswhe 1791. 
boloiden und den entsprechenden Rotationsfächen, dem hyperbolischen Paraboloid, dem hyperbolischen Cylinder und dem Kegel zu beschäftigen.

Betrachten wir zuerst den Kegel.

Die Asymptotenrichtungen irgend eines hyperbolischen Schnittes eines Kegels zweiten Grades sind bekanntlich diejenigen Erzengenden des Kegels, welche zu dor schneidenden Ebene. parallel sind; dieselben müssen für den in Rede stehenden Fall der gleichseitigen Hyperbel zueinander normal sein. Es handelt sich somit um die Ermittlung derjenigen Durchmesserebenen eines Kegels, welche denselben in Paren von zueinander normalen Erzeugenden schneiden. Zu jeder derartigen Durchmesserebene gebört eine Parallelschar von Ebenen, welche den Kegel nach gleichseitigen Hyperbeln schneiden.

Da jeder beliebige Kegel zweiten Grades als ein Kreiskegel dargestellt werden kann, dessen Basis irgend ein Kreisschnitt jenes Kegels ist, so können wir uns, ohne der Allgemeinheit zu schaden, auf die Besprechung des Kreiskegels beschränken.

\section{Der schiefe Kreiskegel.}

Wir bringen denselben in möglichst einfache Lage zu den Projectionsebenen, indem wir den Basiskreis $K$ in $P_{I}$ und die zu demselben normale Hauptebene in $P_{\mathrm{II}}$ verlegen. Dann seien $A S$ und $B S$ die längste und die kiirzeste Erzeugende, welche am Scheitel den Winkel a miteinander einschliefen, $A S B$ kurz das charakteristische Dreieck des schiefen Kreiskegels, $S M$ die Mittellinie $m$ and $S S^{\prime}$ die Höhe $h$; terner seien $a, b$, $c$ die drei Halbachsen, w. zw. $a$ und $b$ die in $P_{\text {II }}$ liegenden ( $a$ innerhalb des Kegels). und $c$ die auf $P_{\mathrm{II}}$ normale.

Mit Rücksicht auf die Größe des Winkels $\alpha$ wollen wir die drei Falle unterscheiden:

1. $\alpha=90^{\circ}$. Dass in diesem Falle anber der Hauptebene $P_{\mathrm{II}}$ auch die beiden durch $m$ und $h$ gelegten zu $P_{\mathrm{II}}$ normalen Ebenen den Kegel $S K^{1}$ ) nach Erzeugenden schneiden, die aufeinander normal stehen, ist obneweiters Klar, da die Schnittdreiecke der letzten zwei Ebenen mit dem Kegel gleichschenklig-rechtwinklig sind. Es schneidet aber auch jede beliebige durch $m$ und jede beliebige durch $h$ gelegte Ebene aus dem Kegel zwei zueinander normale Erzeugende aus; dies zeigt die Betrachtung der entstandenen rechtwinkeligen Schnittdreiecke, von denen sich jedes der ersten

1) Der Kegel mit dem Scheitel $S$ und dem Basiskreise $K$ wird kurz mit $S K$ bereichnet. 
Gruppe aus zwei gleichschenkligen Dreiecken mit dem gemeinsamen Schenkel $m$ und jedes der zweiten Gruppe aus zwei rechtwinkligen Dreiecken mit $h$ als gemeinsamer Kathete zusammensetzt. Noch leichter wird dies erkannt, wenn man sich über dem Basiskreise $K$ des Kegels die Halbkugel construiert denkt, aut welcher der Kegeischeitel $S$ liegt. Jede durch $S$ und den Mittelpunkt des Basiskreises (gleichzeitig Kugelmittelpunkt $M$ ), also durch $m$, ebenso jede durch $S$ normal zu $P_{\mathrm{I}}$, also durch $h$, gelegte Ebene schneidet die Halbkugel nach einem Halbkreise, somit den eingeschriebenen Kegel nach einem bei $S$ rechtwinkligen Dreiecke.

Es gilt somit der Satz:

Jeder schiefe Kreiskegel, in welchem der Winkel am Scheitel des charakteristischen Dreieckes $90^{\circ}$ beträgt, wird durch unendlich viele Parallelscharen von Ebenen nach gleichseitigen Hyperbeln geschnitten. $\left.{ }^{1}\right)$ Alle zu denselben parallelen Durchmesserebenen des Kegels erfüllen zwei Ebenenbüschel, deren Achsen die Mittellinien $m$ und die Höhe $h$ des Kegels sind.

Da durch jede Erzengende des Kegels $S K$ eine Ebene des Büschels $m$ and eine des Büschels $h$ hindurchgeht, so gibt es zu jeder Erzeugenden zwei andere, die auf ihr normal stehen. Ausgenommen sind die Erzeugenden $A S$ und $B S$, für welche die entsprechenden Ebenen der beiden Büschel zusammenfallen.

2. $\alpha>90^{\circ}$. Die Möglichkeit von Durchmesserebenen, welche den Kegel nach zueinander normalen Erzeugenden schneiden, ist sofort einzusehen; denn die darch $S$ gehende Normalebene irgend einer Eirzeugenden schneidet in diesem Falle den Kegel stets nach zwei Erzengenden, deren jede auf der ersteren normal steht, daher mit derselben eine Durchmesserebene von der verlangten Eigenschaft bestimmt. Alle jene Normalebenen hüllen einen Kegel, den Normal- oder Reciprocalkegel, ein, dessen Tangierungrsebenen zur Construction der verlangten Durchmesserebenen benützt werden können. ${ }^{2}$ ). Hier soll jedoch ein anderer Weg eingeschlagen werden, indem wir die letztgenannten Ebenen mittelst ihrer Enveloppe direct ermitteln.

Wir construieren über dem Basiskreise $K$ des gegebenen Kegels das Kugelsegment $k$ (Fig. 1), welches den Kegelscheitel $S$ enthält.

Jede Ebene, durch welche dieses Kugelsegment nach einem Halbkreise geschnitten wird, schneidet den Kegel offenbar nach zwei zueinander normalen Erzeugenden. Der in $K\left(P_{\mathrm{I}}\right)$ gelegene Durchmesser eines jeden solchen Kreises halbiert die durch $S$ normal zu jenem Durchmesser gelegte Kugelsehne. Nun liegen die

1) Vergl. G. W eyer, a. a. O, pag. 141, wo in diesem Falle nur von zwei Systemen von Schnittebenen gesprochen wird.

2) Vergl. Peschka, Darst. und proj. Geometrie, III. Ba., pag. 59.

Monatsh. f. Mathematik u. Physik. VII. Jahrg. 
Endpunkte aller durch $S$ gehenden Kugelsehnen, welche durch $P_{I}$ halbiert werden, auf demjenigen Kugelkreise $x\left(X Y=x^{\prime \prime}\right)$, dessen Ebene zu $P_{\text {I }}$ parallel ist und von $S$ die Entfernung $2 h$ besitzt. (Dann ist $S C=C X, S D=D Y$ u. s. w.) Die Mittelpunkte dieser Sehnen erfüllen den Kreis $K_{1}$ in $P_{\mathrm{I}}\left(C D=\frac{1}{2} X Y\right)$. Jede Ebene $\varepsilon$,

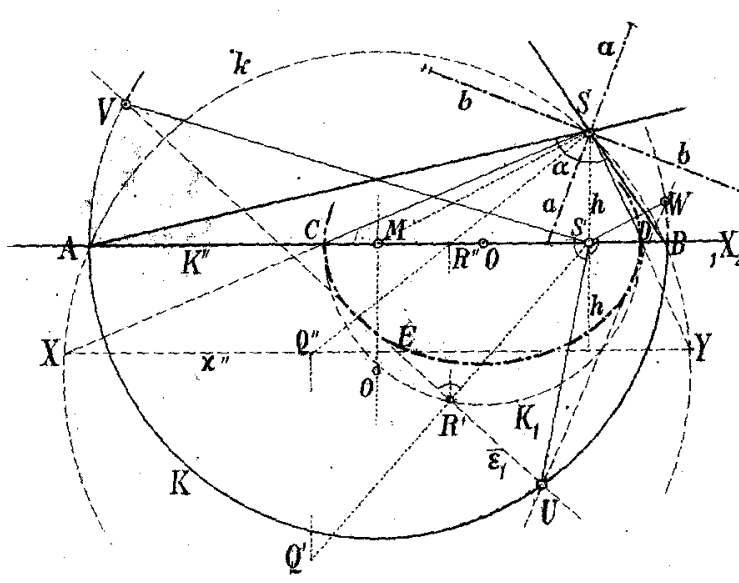

Fig. 1.

welche durch eine solche Sehne, z. B. $S Q=2 S R$, derart gelegt wird, dass ihre erste Spur $\hat{\varepsilon}_{1}$ auf ihr normal steht $\left(\varepsilon_{1}\right.$ 立 $\left.S^{\prime} R^{\prime}\right)$ schneidet das Kugelsegment $k$ nach einem Halbkreise und den Kegel $S K$ nach einem über dem Durchmesser $U V$ des Halbkreises errichteten, bei $S$ rechtwinkligen Dreiecke $U S V$.

Aus der Construction $U V \perp S^{\prime} R^{\prime}$ folgt, dass alle derartigen

Sehnen $U V$ des Basiskreises $K$ des gegebenen Kegels eine Ellipse $E$ einhüllen, für welche der Durchmesser $C D$ des Kreises $K_{1}$ (des Scheitelkreises von $E$ ) die Hauptachse und die Projection $S^{\prime}$ des Kegelscheitels ein Brennpunkt ist. Beachten wir noch, dass wegen der Gleichheit der Peripheriewinkel $A S X$ und $B S Y$ im Kreise $k$ die Winkel $A S B$ und $X S Y$ der Contourerzeugenden der beiden Kegel $S K$ und $S E$ die nämliche Halbierende $a$ besitzen, so erhalten wir folgenden Satz ïber das Kugelsegment:

Alle Ebenen, welche durch einen Pankt eines Kugelsegmentes, das kleiner als eine Halbkugel ist, hindurchgehen, und, dasselbe nach Halbkreisen schneiden, hüllen einen elliptischen Kegel ein, dessen Achsenrichtungen mit denen der gleichartigen (inneren und äuberen) Achsen desjenigen Kegels zusammenfallen, der den gegebenen Punkt zum Scheitel und den Grenzkreis des Kugelsegmentes zur Basis hat.

Mit Rücksicht auf die Bedeutung der Halbkreisschnittebenen des Kugelsegmentes für den Kegel $S K$ können wir den vorigen Satz über das Kugelsegment ohneweiters auch für den schiefen Kreiskegel verwenden und ihn folgendermaßen ausdrücken:

Alle Durchmesserebenen eines schiefen Kreis. kegels mit dem Winkel $\alpha>90^{\circ}$, welche denselben nach Erzeugenden sehneiden, die zu einander normal 
sind, hällen einen elliptischen Kegel ein, dessen Achsenrichtungen mit denen der gleichartigen (inneren und äuberen) Achsen des gegebenen Kegels zusammenfallen.

Jede Sehne $(U V, L W)$ des Kreises $K$, wolche gleichzeitig Tangente der Ellipse $E$ ist, bestimmt mit $S$ eine Tangentialebene des Kegels $S E$, somit eine Durchmesserebene des Kegels $S K$ mit zwei zueinander normalen Schnitterzeugenden; diese selbst sind die Verbindungsgeraden des Kegelscheitels mit den Endpunkten der betreffenden Sehne $(S U, S V$ und $S U, S W$ ).

Jede zu irgend einer Tangentialebene des Kegels $S E$ parallele Ebene schneidet den Kegel $S K$ nach einer gleichseitigen Hyperbel.

Es wird somit auch jeder schiefe Kreiskegel mit dem Winkel $\alpha>90^{\circ}$ von unendlich vielen Parallelscharen von Ebenen, deren Stellungen durch die Tangentialebenen des Hilfskegels $S E$ bestimmt sind, nach gleichseitigen Hyperbeln geschnitten.

3. $\alpha<90^{\circ}$. In diesem Falle lässt nicht jeder schiefe Kreiskegel gleichseitige Hyperbelschnitte zu; die Möglichkeit solcher Schnitte ist an eine bestimmte Beziehung zwischen den Größen $a, h$ und dem Radius $r$ des Basiskreises $\hat{K}$ gebunden. $\left.{ }^{1}\right)$

Die construetive Untersuchung irgend eines vorliegenden Falles erfolgt mit Benützung der dem Kegel umgeschriebenen $\mathrm{K} \mathrm{u} g \mathrm{el} k$ äuberst einfach; das Segment, in welchem der Kegel liegt, ist hier größer als eine Halbkugel.

Die zu $P_{\text {I }}$ parallele Ebene im Abstande $2 h$ vom Kegelscheitel $\$$ (Fig. 2) schneidet das untere Ergänzungssegment nach einem Kreise $x\left(X Y=x^{\prime \prime}\right)$, wenn die $\mathrm{Höhe} h$ des Kegels kleiner ist als die Höhe $M N$ des Erg änzungssegmentes. Der Kugelkreis $x$ bestimmt wie trüher mit $S$ den Kegel, dessen Erzengenden durch $P_{\mathrm{I}}$ in den Punkten des Kreises $K_{1}$ halbiert werden, und die Ebenen, welche durch diese Erzeugenden so gelegt werden, dass ihre ersten Spuren auf denselben normal

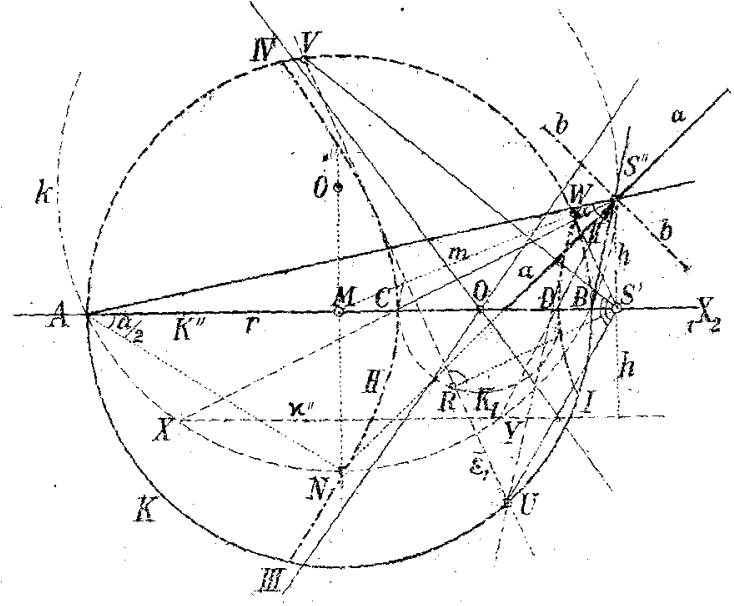

Fig. 2.

1) Vergl. G. W e y er, a. a. O, pag. 143 , wonach $x$ nieht unter $90^{\circ}$ sein dürfte. 
stehen, schneiden die Kugelsegmente nach Halbkreisen and den gegebenen Kegel $S K$ nach zueinander normalen Erzeugenden. Die ersten Spuren dieser Ebenen hüllen jedoch in diesem Falle, da $S^{\prime}$ außerhalb des Kreises $K_{1}$ liegt, eine Hyperbel $H$ ein; dieselbe ist die Schnittlinie von $P_{I}$ nait der Enveloppe jener Ebenen, einem elliptischen Kegel, dessen innere Achse mit der äuheren $b$. des gegebenen Kegels zusammenfällt.

Es gelten somit in Betreff der Ebenen, welche ein Kugelsegment, das größer als eine Halbkugel ist, nach Halbkreisen und einen schiefen Kreiskegelmit dem Winkel $a<90^{\circ}$ nach zueinander normalen Erzeugenden, bezw. nach gleichseitigen Hyperbeln, schneiden, für den Fall, dass die Höhe $h$ des Kegels kleiner ist als die Höhe $M N$ des Ergänzungssegmentes, die den früheren Sätzen analogen, wobei jedoch die Achsen des Hilfskegels in die Richtungen der ungleichartigen des gegebenen fallen.

Nicht zu jeder Erzeugenden des Kegels $S K$ gehören hier zwei zu derselben normale Erzeugenden wie in den beiden früheren Fällen, sondern nur zu denjenigen, deren Fubpunkte auf den zwischen den beiden Hyperbelästen gelegenen Kreisbogen. I III and II IV von $K$ liegen, denn nur für solehe Punkte ist es möglich, an die Hyperbel zwei Tangenten, somit durch die entsprechenden Kegelerzengenden $\mathrm{zw}$ ei Tangentialebenen an den Hilfskegel $S H$ zu legen. Zu jeder der vier Erzeugenden SI - SIV gehört nur eine normale Erzengende, nämlich diejenige, deren Fufpunkt der zweite Schnittpunkt der Tangente des betreffenden Hyperbelpunktes mit dem Basiskreise $K$ des gegebenen Kegels ist.

Die Bedingung dafür, dass der schiefe Kreiskegel mit dem Winkel $\alpha<90^{\circ}$ durch unzählig viele Parallelscharen von Ebenen nach gleichseitigen Hyperbeln geschnitten werden könne, ist nach dem Obigen $\dot{h}<M N$ oder mit Rücksicht auf $M N=r \operatorname{tg} \frac{a}{2}$ $h<r \operatorname{tg} \frac{\alpha}{2}$.

Ist $h=r \operatorname{tg} \frac{\alpha}{2}$, dann berührt die im Abstande $2 h$ von $S$ za $P_{\mathrm{I}}$ parallele Ebene die Kugel im Punkte $N$; es gibt dann nur eine einzige Ebene, die durch die innere Achse $\alpha$ gehende zu. $P_{\text {II }}$ normale Hauptebene $(a c)$, welche den Kegel nach zwei zueinander normalen Erzeugenden schneidet, somit auch nur eine einzige Parallelschar gleichseitiger Hyperbelschnitte.

Ist endlich $h>r \operatorname{tg} \frac{\alpha}{2}$, dann kann der schiefe Kreiskegel nach gleichseitigen Hyperbeln nicht geschnitten werden.

Im letzteren Falle ist der Winkel, welchen die in der Hauptebene ( $a c$ ) gelegenen Erzeugenden miteinander einschließen, 
$\beta<90^{\circ}$, während in dem unmittelbar vorhergehenden $\beta=90^{\circ}$ und in allen früheren Fällen $\beta>90^{\circ}$ ist.

Daraus ist $\mathrm{zu}$ erkennen, dass nicht der Winkel $\alpha$ derjenigen Erzeugenden, die in der Hauptebene $(a b)$ liegen, auf der die Kreisschnittebenen normal stehen, für die Möglichkeit gleichseitighyperbolischer Sehnitte eines Kegels zweiten Grades mafogebend ist, sondern der Winkel $\beta$, der von den Erzeugenden eingeschlossen wird, die in der Hauptebene (ac) liegen; und zwar gibt es keine, eine oder unendlich viele Parallelscharenderartiger Sehnittebenen, $j$ e nachden $\beta \leqq 90^{\circ}$ ist.

Es kann das oben in dem besonderen Falle $\alpha<90^{\circ}$ für die Möglichkeit gleichseitig-hyperbolischer Schnitte aufgestellte Kriterium $h \leqq r \operatorname{tg} \frac{\alpha}{2}$ für jeden beliebigen Kreiskegel benützt werden; es ist allgemein giltig.

\section{Der Rotationskegel.}

Bezeichnen wir den Winkel der beiden in einem Achsenschnitte gelegenen Erzeugenden mit $\alpha$, so ist ohneweiters klar, dass es tür $\alpha<90^{\circ} \mathrm{keine}$ gleichseitigen Hyperbelschnitte gibt. Hingegen existieren deren unendlich viele für $\alpha=90^{\circ}$ und $a>90^{\circ}$, und zwar sind die Parallelscharen der betreffenden Schnittebenen im ersten dieser zwei Fälle zur Rotationsachse parallel, im zweiten zu derselben geneigt.

Im letzten Falle ist die Stellung jener Parallelscharen wieder durch die Ebenen bestimmt, welche den Rotationskegel nach zueinander normalen Erzengenden schneiden. Dieselben hüllen einen mit dem gegebenen coachsialen Rotationskegel ein, dessen Basisradius $\rho$ aus der Höhe $h$ und dem Basisradius $r$ des gegebenen Kegels mittelst der Gleichung $2 p^{2}=r^{2}-h^{21}$ ) leicht construiert werden kann.

Am einfachsten wird jedoch jener Hilfskegel auf constructivem Wege erhalten, indem man in analoger Weise wie früher beim schiefen Kreiskegel die dem Rotationskegel amgeschriebene $\mathrm{Ku}$ gel benützt.

\section{Das zweischalige dreiachsige Hyperboloid.}

Da ein Hyperboloid und sein Asymptotenkegel von einer Ebene nach ähnlichen oder conjugiert ähnlichen Kegelschnitten geschnitten werden, gibt jede Ebene, welche den Asymptotenkegel nach einer gleichseitigen Hyperbel schneidet, einen ebensolchen

1) Diese einfache Beziehung zwischen $\rho, r$ und $h$ wird durch eine kleine Rechnung aus der entsprechenden Figur erhalten.

Der Winkel $\omega$ eines Achsenschnittes des Filfskegels ist durch die Gleichung

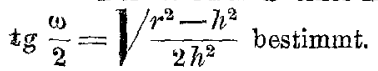


Schnitt mit dem Hyperboloid. Somit ist die Untersuchung über die Möglichkeit gleichseitig-hyperbolischer Schnitte eines Hyperboloides zurückgeführt auf die analoge Untersuchung des zugehörigen As y mptoten kegels; dies erfolgt mit Benützung der Kreisschnitte desselben in der beim Kegel besprochenen Weise.

Beachtet man den Zusammenhang zwischen den. Winkeln der. in den Hauptebenen gelegenen Erzeugenden des Asymptotenkegels (den Asymptoten der beiden hyperbolischen Hauptschnitte des. Hyperboloides) and den Achsen desselben, so folgt mit Rücksicht. auf das boim Kegel über den Einfluss der beiden Winkel $\alpha$ und $\beta$ Gesagte, dass das zweischalige Hyperboloid nur dann nach gleichseitigen Hyperbeln geschnitten werden. kann, wenn die reelle Achse nicht die gröbte der drei Achsen ist.

Bringen wir den Asymptotenkegel in dieselbe Lage wie den schiefen Kreiskegel in Fig. 1 und 2, und behalten wir dieselbe Bezeichnung bei, dann ist für $a>90^{\circ}$ die reelle Achse $2 a$ des Hyperboloides die kleinste $(a<b<c)$, für $a<90^{\circ}$ hingegen die imaginäre $2 b \quad(b<a<c)$; für $\alpha=90^{\circ}$ sind die reelle Achse $2 a$ and die imaginäre $2 b$ einander gleich, jedoch beide kleiner als die imaginäre $2 c(a=b, b<c)$.

Es fällt daher die Richtung der inneren Achse des. elliptischen Hilfskegels, welcher von denjenigen Ebenen eingehüllt wird, die den Asymptotenkegel nach zueinander normalen Erzeugenden schneiden und somit die Stellung der Parallelscharen von Ebenen bestimmen, welche das Hyperboloid nach gleichseitigen Hyperbeln schneiden, mit jener der kleinsten Achse des Hyperboloides zusamen.

Im Falle $a=90^{\circ}$, wo es nicht eine, sondern zwei kleinste Achsen gibt, geht jener Hilfskegel in eine mit der Hauptebene $(a b)$ zusammenfallende Ebene über, welche von denjenigen Durchmessern des Eyperboloides begrenzt wird, die den beiden Kreisschnittsystemen conjugiert sind; die Tangentialebenen jenes Hilfskegels bilden dann die zwei Ebenenbïschel, deren Achsen die genannten zwei Durchmesser sind.

Aus dem Vorangehenden ergibt sich auch der Satz, dass alle Ebenen, welche durch einen Punkt gehen und das Hyperboloid nach gleich seitigen Hyperbeln sehneiden (die Möglichkeit solcher Schnitte rorausgesetzt), einen elliptischen Kegel (den Parallelkegel unseres Hilfskegels) einhüllen, dessen innere Achse mit der kleinstendes Hyperboloides parallel ist ${ }^{1}$ ).

1) Dieser Satz, der natürlich auch für den Kegel und, was sich aus dem Folgenden ergeben wird, für die übrigen hier in Frage kommenden Flächen. zweiten Grades gilt, wurde von Schlömileh am Schlusse seiner eingangs erwähnten analytischen Abhandlung angeführt; jener Kegel, bezw. seine Bestimmungselemente, wurden jedoch dort nicht näher besprochen. 
Es ergibt sich auch weiter, dass in dem besonderen Falle, wo $a=b$ ist, alle diese Ebenen zwei Büschel bilden, deren Achsen dureh jenen Punkt gehen und zu den den Kreisschnittebenen conjugierten Durchmessern des Hyperboloides parallel sind.

\section{Das zweischalige Rotationshyperboloid.}

Dasselbe kann nur dann nach gleichseitigen Hyperbeln gesehnitten werden, wenn die Erzeugenden eines Achsenschnittes des Asymptotenkegels einen Winkel einschließen, der nicht kleiner" als $90^{\circ}$ ist. Dies tritt dann ein, wenn die reelle (Rotations-) Achse des Hyperboloides nicht gröber ist als die imaginären Achsen.

Für $a=b=c,\left(\alpha=90^{\circ}\right)$, ist das Hyperboloid gleichseitig; jede zur Rotationsachse parallele Ebene schneidet es nach einer gleichseitigen Hyperbel.

Ist $a<b, b=c,\left(\alpha>90^{\circ}\right)$, dann gibt es unendlich viele gegen die Rotationsachse geneigte Durchmesserebenen und deren Parallelebenen, welche gleichseitige Hyperbelschnitte liefern. Der Radius $p$ des Parallelkreises des von jenen Durchmesserebenen eingehüllten Rotationskegels ist im Abstande $a$ vom Scheitel durch die Gleichung $2 p^{2}=b^{2}-a^{2}$ bestimmt. ${ }^{1}$ )

\section{Das einschalige dreiachsige Hyperboloid.}

Es ist wohl sofort klar, dass hier gleichseitige Hyper. belschnitte nur dann möglich sind, wenn die imaginäre Achse nicht die längste der drei Achsen ist.

Mit Rücksicht auf die Beantwortung einer Frage, die weiter unten über das einschalige Hyperboloid, dasselbe als Regelfäche betrachtet, gestellt wird, sollen die einzelnen durch die verschiedenen Gröbenverhältnisse der drei Achsen bedingten Fälle kurz besprochen werden, wobei wir die aus der Betrachtung des schiefen Kreiskegels gewonnenen Resultate benützen.

Ist $2 a$ die imaginäre Achse, so liegen die beiden hyperbolischen Hauptschnitte der Fläche in den Hauptebenen $(a b)$ und $(a c)$; die Winkel der in den letzteren gelegenen Erzengenden des Asymptotenkegels (oder die äuferen Winkel der Asymptoten jener zwei Hauptschnitte) seien $a$, bezw. $\beta$.

I) Der Winkel $\omega$ des Achsenschnittes dieses Rotationskegels oder der Neigrngswinkel $\frac{\omega}{2}$ jener Durchmesserebenen mit der Rotationsachse ergibt sich wie oben beim Kegel aus tg $\frac{\omega}{2}=\sqrt{\frac{b^{2}-a^{2}}{2 a^{2}}}$. 
1. $a>b>c ;\left(\alpha<90^{\circ}, \beta<90^{\circ}\right)$. Die imaginäre Achse ist die längste. Es sind keine gleichseitigen Hyperbelschnitte möglich.

2. $\left.a=c, a>b^{1}\right) .\left(\alpha<90^{\circ}, \beta=90^{\circ}\right.$. Nur der Hauptschnitt (ac) und die zu demselben parallelen Schnitte sind gleichseitige Hyperbeln.

3. $a=b, \quad a<c ; \quad\left(\alpha=90^{\circ}, \quad \beta>90^{\circ}\right)$. In diesem Falle schneiden die Hauptebene $(a b)$ und unendlich viele andere Durchmesserebenen sammt ihren Parallelebenen das Hyperboloid nach gleichseitigen Hyperbeln. Jene Durchmesserebenen erfüllen zwei Ebenenbüschel, deren Achsen die zwei den Kreisschnittebenen des Hyperboloides conjugierten Durchmesser $d_{1}$ und $d_{2}$ sind (Fig. 3).

4. $a<b<c ;\left(\alpha>90^{\circ}, \beta>90^{\circ}\right)$. Die imaginäre Achse ist die kleinste. Alle unendlich vielen Durchmesserebenen mit gleichseitigen Hyperbelschnitten "hüllen einen elliptischen Kegel ein, dessen innere Achse mit der imaginären Achse des Hyperboloides zusammenfällt.

5. $b<a<c ;\left(\alpha<90^{\circ}, \beta>90^{\circ}\right)$. Die kleinste Achse ist die reelle $2 b$. Alle unendich vielen Durchmesserebenen mit gleichseitigen Hyperbelschnitten hüllen in diesem Falle einen elliptischen Kegel ein, dessen innere Achse mit der kleineren (2b) der beiden reellen Achsen des Hyperboloides zusammenfällt.

Die Eigenschaft, dass das einschalige Hyperboloid eine Regelfläche ist, durch deren Punkte je zwei Erzengende hindurchgehen, führt uns unwillkürlich zu der Frage nach dem Orte derjenigen Punkte des Hyperboloides, in denen die beiden Erzeugenden zueinander normal sind.

In jeder Parallelschar von Ebenen, welche das einschalige oder windschiefe Hyperboloid nach gleichseitigen Hyperbeln schneiden, befinden sich auch zwei Berührungsebenen desselben; die durch ihre Berührungspunkte hindurchgehenden Erzengendenpaare sind degenerierte gleichseitige Hyperbeln, sie stehen auf einander normal. Es sind dies diejenigen Erzeugenden des Hyperboloides, welche zu den Erzeugenden des Asymptotenkegels parallel sind, die in der jener Parallelschar entsprcchenden Durchmesserebene liegen.

Aus dieser Betrachtung ergibt sich der folgende Satz:

Alle Berührungsebenen eines windschiefen Hyperboloides, welche zu denjenigen Durchmesserebenen desselben und seines Asymptotenkegels parallel sind, die den letzteren nach zueinander normalen Erzeugenden schneiden, hüllen eine developpable Fläche vierter Classe

1) Der Fall, wo die beiden reellen Halbachsen $b$ und $c$ miteinander vertauscht erscheinen, soll weder hier, noch in den übrigen Fällen eigens erwähnt werden, denn er ergibt nichts Neues; es erscheint nur das Hyperboloid $\mathrm{um}$ die imaginäre Achse um $90^{\circ}$ gedreht. 
ein, welche dem Hyperboloide umgeschrieben ist und den von jenen Durchmesserebenen eingehüllten elliptischen Kegel zum Richtkegel hat. Diese Dereloppable $D$ berührt das Hyperboloid nach einer Curve vierter Ordnung $C_{4}$, welche der Ort derjenigen Punkte des Hyperboloides ist, in denen die Erzeugenden zueinander normal sind.

Da das Hyperboloid und der Richtkegel alle drei Hauptebenen gemeinsam haben, sind dieselben für die $C_{4}$ Ebenen orthogonaler Symmetrie; daher sind die Projectionen der $C_{4}$ auf alle drei Hauptebenen Kegelschnitte, deren Achsen mit denen der entsprechenden Hauptschnitte der Lage nach zusammenfallen.

Infolge der dreifachen orthogonalen Symmetrie ist die $C_{4}$ für den Mittelpunkt $M$ des Hyperboloides als Centrum auch centrisch symmetrisch.

Die construetive Ermittlung der $C_{4}$ erfolgt am besten in der Weise, dass man sie nicht als Berührungscurve des Hyperboloides mit der Developpabeln, sondern als Durchdringungscurve desselben mit einem der drei ihr doppelt umschriebenen Cylinder (welche die $C_{4}$ auf die Hauptebene als Kegelschnitte projicieren) oder mit dem ihr aus $M$, dem im Endlichen gelegenen Eckpunkte des gemeinsamen Poltetraeders, doppelt umschriebenen Kegel (welcher die oben hervorgehobene centrische Symmetrie der $C_{4}$ bestimmt) construiert. Die in den Hauptebenen gelegenen Erzeugenden des letzteren werden als die Verbindungsgeraden des Mittelpunktes $M$ mit denjenigen Punkten der $C_{4}$, die in den Hauptschnitten des Hyperboloides liegen, leicht erhalten; diese Punkte sind aber die Berührungspunkte der Hauptschnitte mit denjenigen Tangenten, welche zu den in den gleichnamigen Hauptebenen gelegenen Erzeugenden des von den wiederholt erwähnten Durchmesserebenen des Asymptotenkegels eingehüllten Hilfskegels parallel sind.

In den einzelnen, oben besprochenen fünf Fällen des einschaligen Hyperboloides ist uiber den Ort der Punkte mit zueinandernormalen Erzeugenden Folgendes zu bemerken:

1. Das Hyperboloid enthält keine solchen Punkte.

2. Es gibt nur zwei solche Punkte, nämlich die Endpunkte $B_{1}$ und $B_{2}$ der zum gleichseitig-hyperbolischen Hauptschnitte $(a c)$ normalen Achse $2 b$.

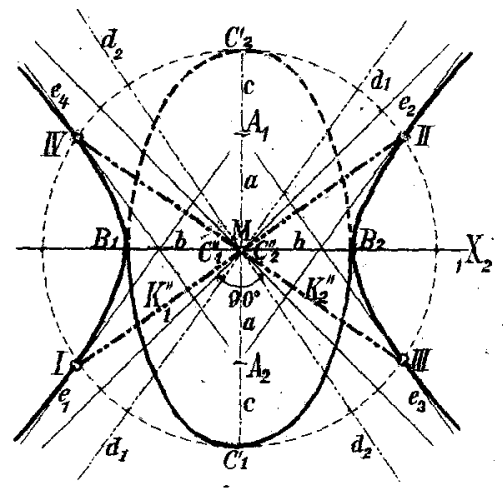

Fig. 3. 
In den übrigen drei Fälen gibt es jederzeit unendlich viele Punkte mit zueinander normalen Erzeugenden.

3. Die Developpable $D$ zerfällt in zwoi Cylinderflächen zweiten Grades, deren Achsen $d_{1}$ und $d_{2}$ (Fig. 3) die den Kreisschnittebenen des Hyperboloides conjugierten Durchmesser desselben sind. Die Berührungscurve $C_{4}$ besteht aus den beiden Durchmesser-Kreisschnitten $K_{1}$ and $K_{2}$ des Hyperboloides.

4. Die Developpable $D$ berührt das Hyperboloid in keinem Punkte der Kehlellipse. Deshalb schneidet die $C_{4}$ die Kehlellipse nicht, sondern sie besteht aus zwei durch die Ebene der letzteren voneinander getrennten, geschlossenen $\mathrm{Z}$ weigen. Die Projection derselben auf die Hauptebene $(a b)$ ist eine Hyperbel, deren reelle Achse mit der imaginären des Hyperboloides zusammenfällt, jene auf die Hauptebene $(a c)$ ist eine Ellipse mit parasitischen Theilen innerhalb jedes der beiden Äste dieses hyperbolisehen Hauptschnittes, und jene auf die Hauptebene $(b c)$ ist eine Ellipse, welche die Kehlellipse einschliebt.

5. Die Developpable $D$ berührt das Hyperboloid in vier Punkten der Kehlellipse. Deshalb sehneidet die $C_{4}$ die Kehlellipse in vier Punkten; sie besteht auszwei geschlossenen, durch die Hauptebene (ac) voneinander getrennten $\mathrm{Z}_{w}$ eigen. Die Projection derselben auf die Hauptebene $(a b)$ ist eine Hyperbel, deren imaginäre Achse wit der imaginären des Hyperboloides zusammenfällt; .jene auf die Hauptebene $(a c)$ ist eine Ellipse zwischen den beiden Asten des betreffenden hyperbolischen Hauptschnittes, und jene aut die Hauptebene $(b c)$ eine Ellipse mit parasitischen Theilen innerhalb der Kehlellipse.

In Fig. 4 sind die diesen Fall betreffenden Constructionen für ein Hyperboloid durchgeführt, dessen Hauptebene $(a b)$ mit der zweiten Projectionsebene zusammenfällt, und dessen imaginäre Achse $A_{1} A_{2}$ zur ersten Projectionsebene normal ist.

Mittelst eines Kreisschnittes $K$ des Asymptotenkegels wurde in der früher besprochenen Weise jener elliptische Hilfskegel erhalten, dessen Berührungsebenen das Hyperboloid nach gleichseitigen. Hyperbeln schneiden, und der gleichzeitig der Richtkegel der Developpabeln $D$ ist. Seine innere Achse fallt in die Richtung der reellen $B_{1} B_{2}$ des Hyperboloides; die in der Hauptebene $(a b)$ liegenden Erzeugenden sind $d_{1}$ und $d_{2}$, und die Spureurve in der ersten Projectionsebene ist die Hyperbel $H$. Die im Hauptschnitte (ab) des Hyperboloides gelegenen Punkte I, II, III, IV der $C_{4}$ sind die Berührungspunkte der zu $d_{1}$ und $d_{2}$ paarweise parallelen Erzeugenden $e_{1}, e_{2}, e_{3}, e_{4}$ der Developpabeln mit dieser Hyperbel, and die im Hauptschnitte $(b c)$, der Kehlellipse, gelegenen Punkte V, VI, VII, VIII jene der horizontalen Erzeugenden $e_{5}, e_{6}, e_{7}, e_{8}$ der Developpabeln mit der Kehlellipse. Die entsprechenden 
horizontalen Erzeugenden $\varepsilon_{5}, \varepsilon_{6}$ des Richtkegels werden am einfachsten mittelst der beiden horizontalprojicierenden Durchmesserebenen (Berührungsebenen des Richtkegels) erhalten, welche den Asymptotenkegel. nach rechten Winkeln schneiden; die Hori-

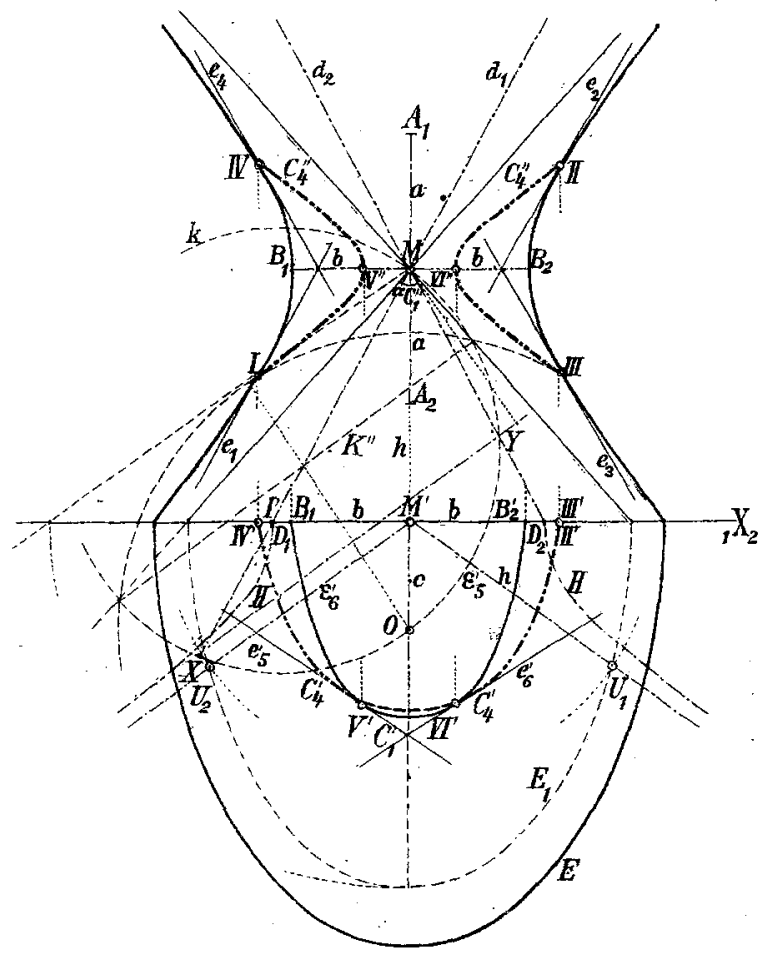

Fig. 4.

zontalspuren dieser zwei Ebenen sind offenbar die Verbindungsgeraden von $M^{\prime}$ mit den Punkten $U_{1}$ and $U_{2}$, in denen die Horizontalspur $E_{1}$ des Asymptotenkegels von dem Kreise mit dem Radius $h\left(M^{\prime} M\right)$ und dem Mittelpunkte $M^{\prime}$ geschnitten wird.

\section{Das einschalige Rotationshyperboloid.}

Für dasselbe liegen die Verhältnisse so wie beim $\mathrm{zwei-}$ schaligen Rotationshyperboloid, es darf nämlich der Winkel des Achsenschnittes des Asymptotenkegels nicht unter $90^{\circ}$ sein. Das ist dann der Fall, wenn die imaginäre (Rotations-)Achse $2 a$ nicht gröber ist als die reellen Achsen.

In Betreff des Ortes der Punkte mit zueinander normalen Erzeugenden ist hier Folgendes zu beachten: 
Ist $a=b=c,\left(\alpha=90^{\circ}\right)$, das einschalige Rotationshyperboloid also gleichseitig, dann ist die demselben umgeschriebene Developpable $D$ ein mit ihm coachsialer Rotationseylinder, und die Berührungscurve mit dem Hyperboloid der Kehlkreis desselben.

Ist $a<b,\left(\alpha>90^{\circ}\right)$ dann zerfällt die Developpable $D$ in zwei mit dem Hyperboloid coachsiale Rotationskegel, welche mit dem von den Durchmesserebenen, die das Hyperboloid nach gleichseitigen Hyperbeln schneiden, eingehüllten Hilfskegel congruent sind. Die Berührungscurve $C_{4}$ zerfällt in zwei zu beiden Seiten des Kehlkreises in gleichen Abständen gelegene Parallelkreise; die constructive Ermittlung derselben bedarf keiner weiteren Erörterung, sie ergibt sich aus dem Früheren.

\section{Das hyperbolische Paraboloid.}

Jede zur Achse des hyperbolischen Paraboloides nichtparallele Ebene schneidet dasselbe nach einer Hyperbel, für welche bekanntlich die Schnittlinien jener Ebene mit den beiden Richtebenen des Paraboloides die Asymptotenrichtungen sind. Im Falle des gleichseitigen Hyperbelschnittes müssen diese zwei Schnittlinien zu einander normal sein. ${ }^{1}$ )

Es handelt sich somit zunächst um die Ermittlung soleher Ebenen, deren Schnittlinien mit zwei sich schneidenden Ebenen zueinander normal sind.

Ist die eine, $g$, der beiden Geraden in der Ebene $\lambda$ gegeben, so schneidet die im Schnittpunkte derselben mit der zweiten Ebene $\mu$ auf $g$ normale Ebene die $\mu$ nach der zu $g$ normalen Geraden $g_{1}$. Und so kann in jedem einzelnen Falle, wenn $\lambda$ und $\mu$ die beiden Richtebenen des hyperbolischen Paraboloides sind, zu einer gegebenen Asymptotenrichtung eines gleichseitigen Hyperbelschnittes die dazugebörige zweite und damit die Stellung der schneidenden Ebene und ihrer Parallelschar gefunden werden.

Wir wollen aber nicht einzelne, sondern wie bei den früher behandelten Flächen die Gesammtheit derartiger Ebenen, welche durch einen Punkt gelegt werden, betrachten.

Es seien $\lambda$ und $\mu$ (Fig. 5) die beiden durch den Scheitel $A$ des hyperbolischen Paraboloides gelegten verticalprojicierenden Richtebenen, die auferdem gegen die erste Projectionsebene $P_{\mathrm{I}}$ gleiche Neigung besitzen; ferner sei der gröhiere der beiden Neigungswinkel der Richtebenen $\left(\alpha>90^{\circ}\right)$ gegen $P_{\mathrm{I}}$ gekehrt. Wir

1) Siehe Pesehka, Darst. und proj. Geom., III. Bd., pag. 172 u. 173. Daselbst wird die Aufgabe gelöst, „durch eine gegebene Gerade eine Ebene zu führen, welche ein hyperbolisches Paraboloid in einer gleichseitigen Hyperbel schneidet". 
nehmen in der Schnittlinie von $\lambda$ und $\mu$ (der Achse des Paraboloides) irgend einen Punkt, z. B. den Scheitel $A$, an und legen durch denselben alle Ebenen, welche $\lambda$ und $\mu$ nach zueinander normalen Geraden schneiden. Zu jeder Geraden $g$ in $\lambda$ gehört nur eine auf ihr normale $g_{1}$ in $\mu$; es bilden somit die Spurpunkte solcher Geradenpaare in $P_{\text {I }}$ zwei projectivische Punktreihen, deren Träger die Horizontalspuren $\overline{\lambda_{1}}$ und $\overline{\mu_{1}}$ der Richtebenen sind. Die Verbindungsgeraden entsprechender Punkte $\left(P P_{1}, Q Q_{1}\right.$ $\left.T T_{1}^{\infty}, U^{\infty} U_{1}, \ldots\right)$ sind die Horizontalspuren der gesuchten Ebenen; sie hüllen die Ellipse $E$ ein, deren große Achse offenbar $T U_{1}$ und deren Mittelpunkt $A^{\prime}$ ist. Die kleine Achse $B_{1} B_{2}$ wird am einfachsten mittelst der gegen $\lambda$ and $\mu$ gleichgeneigten Ebenen erhalten, deren Horizontalspuren $P P_{1}$ und $Q Q_{1}$ zu $T U_{1}$ parallel sind. Die in denselben gelegenen Dreiecke $A P P_{1}$ und $A Q Q_{1}$ sind gleichschenklig - rechtwinklig, daher $A B_{1}=(A) B_{1}$ $=P B_{1}=T A^{\prime}=a$.

Es gilt somit der folgende Satz:

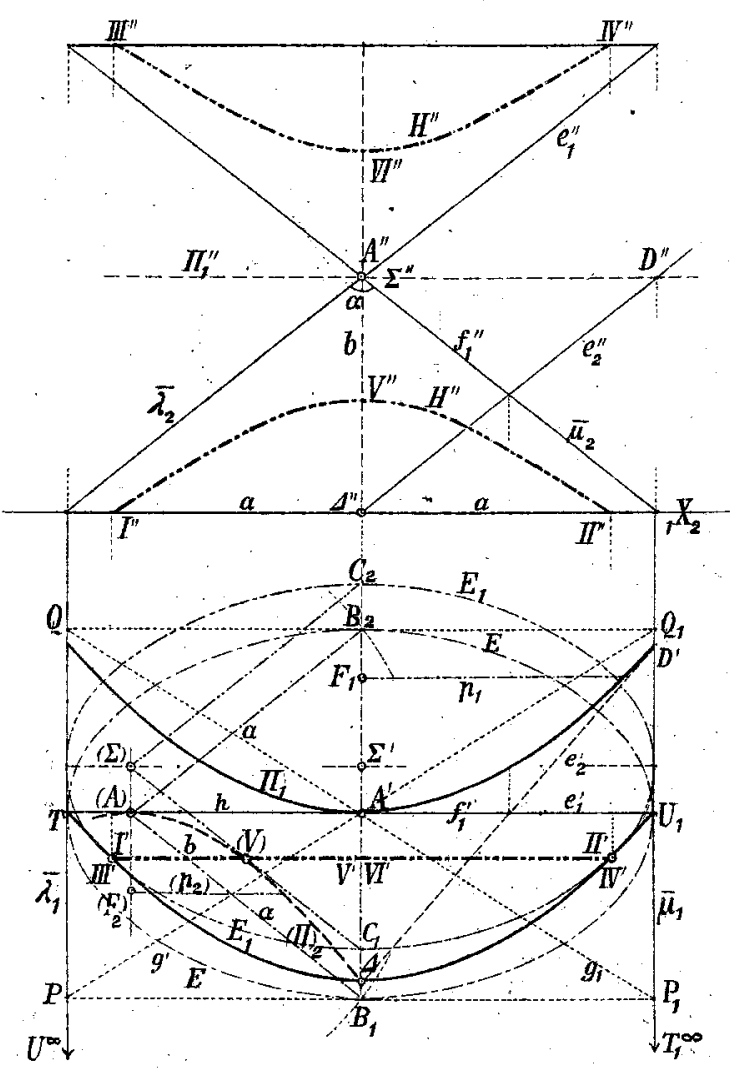

Fig. 5.

Alle Ebenen, welche durch irgend einen Punkt der Schnittlinie zweier sich unter schiefem Winkel schneidenden Ebenen so gelegt werden, dass sie dieselben nach zueinander normalen Geraden schneiden, hüllen einen elliptischen Kegel ein, welcher die gegebenen Ebenen zu Tangentialebenen besitzt, und dessen innere Achse im größeren (stumpfen) Winkelraum derselben liegt und auf ihrer Schnittlinie normal steht. 
Dieser Satz ${ }^{1}$ kann ohneweiters aut diejenigen Ebenen bezogen werden, welche ein hyperbolisches Paraboloid nach gleichseitigen Hyperbeln schneiden. wenn jene zwei Ebenen als die durch die Achse des Paraboloides gelegten Richtebenen desselben angenommen werden.

Hiebei ist jedoch zu beachten, dass unter allen Tangentialebenen des Kegels die beiden Richtebenen Grenzlagen einnehmen, in denen die Schnittcurve aus einer Scheitelerzeugenden und der unendlich fernen Geraden der betreffenden Richtebene besteht.

Stehen die beiden Ebenen $\lambda$ and $\mu$ aufeinander normal, so gehört zu jeder durch einen Punkt $A$ der Schnittlinie gehenden und zu derselben schiefen Geraden in der einen Ebene dieselbe normale in der anderen Ebene, nämlich die Normale in $A$ zur Schnittlinie.

Es erfüllen somit alle Ebenen, welchedurch einen Punkt der Schnittlinie zweier sich unter rechtem Winkel schneidenden Ebenen gehen und dieselben nach zueinander normalen Geraden schneiden, zwei Ebenenbüschel, deren Achsen die in den beiden Ebenen liegenden Normalender Schnittlinie in jenem Punkte sind.

Stellen die Ebenen $\lambda$ und $\mu$ die Richtebenen eines gleichseitigen hyperbolischen Paraboloides ror, so ergibt sich mit Rücksicht darauf, dass die in den Richtebenen liegenden Normalen ihrer Schnittlinie zu den Scheitelerzeugenden parallel sind, aus dem Vorangehenden folgender Satz:

Alle Ebenen, welche durch einen festen Punkt gehen und ein gleichseitiges hyperbolisches Paraboloid nach gleichseitigen Hyperbeln schneiden, erfüllen zwei Ebenenbüschel, deren Achsen zu den Scheitelerzengenden der Fläche parallel sind.

Hiebei stellen wieder die zu den Richtebenen parallelen Ebenen der beiden Büschel Grenzlagen dar, in denen die Schnittcurve aus einer Erzeugenden und der unendlich fernen Geraden der betreffenden Ebene oder der entsprechenden Richtebene besteht.

Aus dem Vorangehenden erhellt auch sofort die Richtigkeit des folgenden Satzes:

1) Beachtet man, dass der im stumpfen Winkel der beiden Richtebenen $\lambda$. und $\mu$ (Fig. 5) liegende Hauptsehnitt $\Pi_{2}$ des hyperbolischen Paraboloides jener mit dem kleineren Parameter $p_{2}$ ist, so ergibt sich daraus der früher erwähnte Schlömilch'sche Satz, nach welchem alle Ebenen, die durch einen festen Punkt gehen and ein hyperbolisches Paraboloid nach gleichseitigen Hyperbeln schneiden, einen elliptischen Kegel einhüillen, dessen Achse zum kleineren Parameter der Fläche parallel ist". ermitteln.

Nach dem Obigen sind die Bestimmungselemente dieses Kegels leicht zu

Aus der Figur ist ersichtlich, dass der Punkt $(A)$ ein Brennpunkt der Basisellipse $E$ des Kegels $S E$ ist; die Excentricität der Ellipse $E$ ist somit gleich der Höhe 7 des Kegels. 
Jedezu einer Scheitelerzeugenden des gleichseitigen hyperbolischen Paraboloides parallele und zur Achse geneigte Ebene schneidet dasselbe nach einer gleichseitigen Hyperbel.

Ist die betreffende Ebene zu beiden Scheitelerzeugenden parallel, so liefert sie einen Normalschnitt zur Achse.

In Betreff des Ortes der Punkte mit zueinander normalen Erzeugenden sind die Verhältnisse beim hyperbolischen Paraboloide viel einfacher als beim windschiefen Hyperboloide. Da der Richtkegel der dem Paraboloide umgeschriebenen Developpabeln $D$ zwei Tangentialebenen besitzt, welche zu den Richtebenen des Paraboloides parallel sind, degeneriert die Developpable $D$ in einen Kegel zweiten Grades $\Sigma E_{1}$ (Fig. 5), welcher mit dem Richtkegel congruent ist. Der Scheitel $\Sigma$ dieses Kegels liegt auf der Achse des Paraboloides und zwar innerhalb des Hauptschnittes $\Pi_{1}$ mit dem größeren Parameter; er wird am einfachsten erhalten, indem man an das Paraboloid, bezw. an den Hauptschnitt $\Pi_{2}$, die Tangentialebene parallel zur Ebene $A P P_{1}$ ( $A E^{\prime}$ als Richtkegel vorausgesetzt) legt. $(\Sigma) C_{1} \|(A) B_{1}$.

Die Berührungscurve des Kegels $\Sigma E_{1}$ mit dem hyperbolischen Paraboloide, d. h. der Ort der Punkte mit zueinander normalen Erzeugenden, ist die Hyperbel $H$, deren Ebene auf der Achse des Paraboloides normal steht, deren Asymptoten somit zu den Scheitelerzeugenden parallel sind; die reelle Achse derselben ist parallel zur inneren Achse des Richtkegels oder zum kleineren Parameter des Paraboloides.

Ist das hyperbolische Paraboloid gleichseitig, dann degeneriert die Developpable $D$ in die Tangentialebene im Scheitel, und der Ort der Punkte mit zueinander normalen Erzeugenden in die beiden Scheitelerzeugenden; es wird somit jede der beiden Scheitelerzeugenden von allen Erzeugenden der anderen Schar rechtwinklig geschnitten, eine Eigenschaft des gleichseitigen hyperbolischen Paraboloides, die bekanntlich unmittelbar aus seiner Entstehung folgt.

\section{Der hyperbolische Cylinder.}

Jede Ebene, die nicht zur Achse des Cylinders parallel ist, schneidet den hyperbolischen Cylinder nach einer Hyperbel, deren Asymptoten die Schnittlinien der Ebene mit den beiden Asymptoten-

Der bei jeder der besprochenen Flächen zweiten Grades erwähnte Hilfs kegel kann bei der Lösung der Aufgabe, durch eine gegebene Gerade Ebenen zu legen, welche eine gegebene Fläche zweiten Grades nach gleichseitigen Hyperbeln schneiden (die Möglichkeit solcher Schnitte vorausgesetzt), mit Vortheil benitzzt werden, indem man irgend einen Punkt dieser Geraden als Scheitel des Kegels wählt. Nach der Anzahl der Tangententialebenen, welche durch die Gerade an den Kegel gelegt werden können, (je nachdem die Gerade außerhalb, auf oder innerhalb der Kegelfläche liegt) hat die genannte Aufgabe zwei, eine oder keine Lösungen. 
ebenen des Cylinders sind; diese Schnittlinien müssen in dem vorliegenden Falle zueinander normal sein.

Es ist leicht einzusehen, dass in Bezug auf die Ebenen, welcheeinen allgemeinen oder einen gleichseitigen hyperbolischen Cylinder nach gleichseitigen Hyperbeln schneiden, die oben für das hyperbolische Paraboloid entwickelten Sätze mit geringen Abänderungen ebenfalls Giltigkeit haben, wenn die Asymptotenebenen des Cylinders an die Stelle der durch die Achse des Paraboloides gelegten Richtebenen $\lambda$ and $\mu$ treten. 